\title{
ON NON-UNIFORM AND GLOBAL DESCRIPTIONS OF THE RATE OF CONVERGENCE OF ASYMPTOTIC EXPANSIONS IN THE CENTRAL LIMIT THEOREM
}

\author{
PETER HALL and T. NAKATA
}

(Received 22 February 1984; revised 10 December 1984)

Communicated by T. C. Brown

\begin{abstract}
The leading term approach to rates of convergence is employed to derive non-uniform and global descriptions of the rate of convergence in the central limit theorem. Both upper and lower bounds are obtained, being of the same order of magnitude, modulo terms of order $n^{-r}$. We are able to derive general results by considering only those expansions with an odd number of terms.
\end{abstract}

1980 Mathematics subject classification (Amer. Math. Soc.): 60 F 05, 60 G 50.

\section{Introduction and results}

Using the leading term approach [1,2] we obtain new bounds to rates of convergence in various metrics. These results are combined to complement earlier work of Heyde and Nakata [3]. By way of notation, let $X, X_{1}, X_{2}, \ldots$ be i.i.d.r.v. with zero mean and unit variance, let $S_{n}=\sum_{j=1}^{n} X_{j}, F_{n}(x)=P\left(S_{n} \leqslant n^{1 / 2} x\right)$, and $(2 \pi)^{1 / 2} \Phi(x)=\int_{-\infty}^{x} e^{-u^{2} / 2} d u$. Assume that $E\left(X^{2 k+2}\right)<\infty$ for integer $k \geqslant 0$, let $\mu_{j}=E\left(X^{j}\right)$ for $1 \leqslant j \leqslant 2 k+2$, define $\mu_{2 k+3}$ arbitrarily, and define cumulants $\kappa_{j}(1 \leqslant j<\infty)$, polynomials $P_{j}$ and $Q_{j}$, leading term function ${ }_{k} L_{n}(x)$ and its order of magnitude

$$
\begin{aligned}
k_{n} \equiv & n^{-k} E\left\{X^{2 k+2} I\left(|X|>n^{1 / 2}\right)\right\}+n^{-(k+1)} E\left\{X^{2 k+4} I\left(|X| \leqslant n^{1 / 2}\right)\right\} \\
& +n^{-(2 k+1) / 2}\left|E\left\{X^{2 k+3} I\left(|X| \leqslant n^{1 / 2}\right)\right\}-\mu_{2 k+3}\right|
\end{aligned}
$$

as in [2].

(C) 1986 Australian Mathematical Society $0263-6115 / 86 \$ A 2.00+0.00$ 
Theorem 1 is a nonuniform description of error in Chebyshev-EdgeworthCramér expansions after the leading term has been accounted for, while Theorem 2 describes order of magnitude of the leading term.

Theorem 1. Assume $E\left(X^{2 k+2}\right)<\infty$ for an integer $k \geqslant 0$, and that Cramér's condition, $\lim \sup _{t \rightarrow \infty}\left|E\left(e^{i t X}\right)\right|<1$, holds. Then

$$
\begin{gathered}
\sup _{-\infty<x<\infty}\left(1+|x|^{2 k+2}\right)\left|F_{n}(x)-\Phi(x)-\phi(x) \sum_{j=1}^{2 k+1} Q_{j}(x) n^{-j / 2}-{ }_{k} L_{n}(x)\right| \\
=O\left(n^{-1 / 2}{ }_{k} \delta_{n}+{ }_{k} \delta_{n}^{2}+n^{-(k+1)}\right) .
\end{gathered}
$$

THeOREM 2. Assume $E\left(X^{2 k+2}\right)<\infty$, and let $E=(0, \varepsilon)$ or $(-\varepsilon, 0)$. Then

$$
\left.\liminf _{n \rightarrow \infty} \sup _{|x| \leqslant \varepsilon}\right|_{k} L_{n}(x) \mid / \delta_{n}, \quad \liminf _{n \rightarrow \infty}\left\{\left.\left.\int_{E}\right|_{k} L_{n}(x)\right|^{p} d x\right\}^{1 / p} /{ }_{k} \delta_{n}
$$

are strictly positive for any $\varepsilon>0$ and $p \geqslant 1$. Moreover, for a constant $C>0$ depending only on $k$, we have $\left.\sup _{-\infty<x<\infty}\left(1+x^{2 k+2}\right)\right|_{k} L_{n}(x) \mid<C_{k} \delta_{n}$.

Theorems 1 and 2 are readily combined to give various descriptions of rates of convergence in nonuniform and $L^{p}$ metrics. For example, we extend a portion of Theorem 1 in [3]:

Corollary. Assume $E\left(X^{2 k+2}\right)<\infty$, Cramér's condition, and $x^{2 k+4} P(|X|>$ $x) \rightarrow \infty$ as $x \rightarrow \infty$. Then for any $p \geqslant 1$ and $r<p(2 k+2)-1$, the ratio

$$
\left\{\int_{-\infty}^{\infty}\left(1+|x|^{r}\right)\left|F_{n}(x)-\Phi(x)-\phi(x) \sum_{j=1}^{2 k+1} Q_{j}(x) n^{-j / 2}\right|^{p} d x\right\}^{1 / p} / k^{\delta_{n}}
$$

is bounded away from zero and infinity as $n \rightarrow \infty$.

\section{Proofs}

We prove only Theorem 1 . The proof of Theorem 2 parallels arguments in [1, Theorems 2.3 and 2.5] and [2]. Symbols $C$ and $p$ denote respectively a generic positive constant and a generic positive integer. 
The characteristic function (Fourier-Stieltjes transform) of

$$
{ }_{k} D_{n}(x) \equiv F_{n}(x)-\Phi(x)-\sum_{j=1}^{2 k+1} P_{j}(-\Phi)(x) n^{-j / 2}-{ }_{k} L_{n}(x)
$$

is given by

$$
\begin{aligned}
{ }_{k} d_{n}(t) \equiv & \alpha^{n}\left(t / n^{1 / 2}\right)-e^{-t^{2} / 2} \sum_{j=0}^{2 k+1} P_{j}(i t) n^{-j / 2} \\
& -n\left\{\alpha\left(t / n^{1 / 2}\right)-\sum_{j=0}^{2 k+3} \mu_{j}\left(i t / n^{1 / 2}\right)^{j} / j !\right\} e^{-t^{2} / 2} .
\end{aligned}
$$

The function $x^{2 k+2}{ }_{k} D_{n}(x)$ has Fourier-Stieltjes transform

$$
\chi(t)=i t(d / d t)^{2 k+2}\left\{(i t)_{k}^{-1} d_{n}(t)\right\} .
$$

Therefore, by [4, Lemma 8 , page 155], we have

$$
\begin{aligned}
& \sup _{-\infty<x<\infty}\left.\left(1+|x|^{2 k+2}\right)\right|_{k} D_{n}(t) \mid \\
& \leqslant C\left\{\left.\int_{0}^{T}\left(t^{-1}+t^{-(2 k+3)}\right)\right|_{k} d_{n}(t) \mid d t\right. \\
&\left.\quad+\left.\sum_{r=0}^{2 k+1} \int_{0}^{T} t^{-(r+1)}\right|_{k} d_{n}^{(2 k+2-r)}(t) \mid d t+T^{-1}\right\}
\end{aligned}
$$

for all $T>1$, provided $\sup _{n, x}\left(1+|x|^{2 k+2}\right)\left|(d / d x)\left\{{ }_{k} D_{n}(x)-F_{n}(x)\right\}\right|<\infty$. The latter inequality follows via a short algebraic argument, using techniques of [1, pages 30-33].

The remainder of the proof consists of estimating the terms on the right hand side of (2.1). We first estimate ${ }_{k} d_{n}^{(l)}(t)=(d / d t)_{k}^{l} d_{n}(t)$ for $t$ in the range $0<t \leqslant n^{\delta}$, and for some $\delta \in\left(0, \frac{1}{2}\right]$. This is carried out in several stages. The following lemma will prove useful. Define

$$
A_{n 1}(t)=\alpha\left(t / n^{1 / 2}\right)-1-\sum_{j=2}^{2 k+3} \mu_{j}\left(i t / n^{1 / 2}\right)^{j} / j !
$$

LEMMA 2.1. The following estimates are valid for all $t>0$, and for all integers $0 \leqslant l \leqslant 2 k+2$ :

$$
\begin{gathered}
\left|A_{n 1}^{(l)}(t)\right| \leqslant C\left(1+t^{2}\right) t^{2 k+2-l} n^{-(k+1)}, \\
n\left|A_{n 1}^{(l)}(t)\right| \leqslant C\left(1+t^{2}\right) t_{k}^{2 k+2-l} \delta_{n} .
\end{gathered}
$$

Furthermore, for each $\varepsilon>0$, there exists $\delta>0$ such that, whenever $0<t \leqslant \delta n^{1 / 2}$, we have

$$
n\left|A_{n 1}\left(t / n^{1 / 2}\right)\right| \leqslant \varepsilon t^{2}
$$


Proof. Since ${ }_{k} \delta_{n}=o\left(n^{-k}\right)$, it suffices to prove (2.3) and (2.4). In (2.3), we shall assume that $l=2 m+1$ is odd; the case of even $l$ may be treated similarly. Now,

$$
\begin{aligned}
& \left|A_{n 1}^{(l)}(t)\right| \leqslant\left|(d / d t)^{l} E\left\{\cos \left(t X / n^{1 / 2}\right)-\sum_{j=0}^{k+1}(-1)^{j}\left(t X / n^{1 / 2}\right)^{2 j} /(2 j) !\right\}\right| \\
& +\mid(d / d t)^{l} E\left\{\sin \left(t X / n^{1 / 2}\right)-\sum_{j=0}^{k}(-1)^{j}\left(t X / n^{1 / 2}\right)^{2 j+1} /(2 j+1) !\right\} \\
& -(d / d t)^{l}(-1)^{k+1} \mu_{2 k+3}\left(t / n^{1 / 2}\right)^{2 k+3} /(2 k+3) ! \\
& \leqslant 2\left[E\left\{\left|X / n^{1 / 2}\right|^{l}\left|t X / n^{1 / 2}\right|^{2(k-m)+3} I\left(|X| \leqslant n^{1 / 2}\right)\right\}\right. \\
& \left.+E\left\{\left|X / n^{1 / 2}\right|^{\prime}\left|t X / n^{1 / 2}\right|^{2(k-m)+1} I\left(|X|>n^{1 / 2}\right)\right\}\right] \\
& +n^{-(2 k+3) / 2}\left|E\left\{X^{2 k+3} I\left(|X| \leqslant n^{1 / 2}\right)\right\}-\mu_{2 k+3}\right| t^{2 k+3-l} \\
& \leqslant 2\left(1+t^{2}\right) t^{2 k+2-l} n^{-1} k_{n},
\end{aligned}
$$

as required for (2.3). Result (2.4) follows from the fact that $\left|\alpha(t)-1+t^{2} / 2\right|=$ $o\left(t^{2}\right)$ as $t \rightarrow 0$.

Choose $\varepsilon>0$ so small that $|\alpha(t)-1| \leqslant 1 / 2$ for $0<t \leqslant 2 \varepsilon$. Define

$$
A_{n 2}(t) \equiv n \log \alpha\left(t / n^{1 / 2}\right)=n \sum_{j=1}^{\infty}(-1)^{j+1}\left\{\alpha\left(t / n^{1 / 2}\right)-1\right\}^{j} / j .
$$

Then for $0<t<\varepsilon n^{1 / 2}$, we have

$$
\begin{aligned}
A_{n 3}(t) & \equiv\left|(d / d t)^{l}\left[A_{n 2}(t)-n \sum_{j=1}^{3 k+3}(-1)^{j+1}\left\{\alpha\left(t / n^{1 / 2}\right)-1\right\}^{j} / j\right]\right| \\
& \leqslant n \sum_{j=3 k+4}^{\infty} j^{-1}\left|(d / d t)^{l}\left\{\alpha\left(t / n^{1 / 2}\right)-1\right\}^{j}\right| .
\end{aligned}
$$

Since $\alpha$ has $2 k+2$ bounded derivatives, and since $|\alpha(t)-1| \leqslant t^{2} / 2$, it follows that for $j \geqslant 3 k+4$ and $0 \leqslant l \leqslant 2 k+2$, we have

$$
\left|(d / d t)^{l}\{\alpha(t)-1\}^{j}\right| \leqslant C j^{l}\left(t^{2} / 2\right)^{k+2}|\alpha(t)-1|^{j-l-(k+2)},
$$

where $C$ does not depend on $j$. Therefore, if $0<t<\varepsilon n^{1 / 2}$, we have (2.5)

$$
A_{n 3}(t) \leqslant C_{1} n n^{-l / 2}\left(t^{2} / 2 n\right)^{k+2} \sum_{j=0}^{\infty}(j+3 k+4)^{l}(1 / 2)^{j} \leqslant C_{2} t^{2(k+2)} n^{-(k+1)} .
$$


Next, observe from (2.2) that

$$
\begin{aligned}
& n^{-1} A_{n 4}(t) \equiv \mid(d / d t)^{t}\left[\sum_{r=2}^{3 k+3}(-1)^{r+1}\left\{\alpha\left(t / n^{1 / 2}\right)-1\right\}^{r} / r\right. \\
&\left.-\sum_{r=2}^{3 k+3}(-1)^{r+1}\left\{\sum_{j=2}^{2 k+3} \mu_{j}\left(i t / n^{1 / 2}\right)^{j} / j !\right\} / r\right] \mid \\
& \leqslant C_{1} \sum_{r=2}^{3 k+3} \sum_{s=1}^{r} \sum_{a=0}^{l}\left|(d / d t)^{a}\left\{A_{n 1}(t)\right\}^{s}\right|\left|(d / d t)^{l-a}\left\{\sum_{j=2}^{2 k+3} \mu_{j}\left(i t / n^{1 / 2}\right)^{j} / j !\right\}^{r-s}\right| \\
& \leqslant C_{2}\left(1+t^{p}\right) t^{2 k+2-l} n^{-(k+2)} .
\end{aligned}
$$

It follows from the definition of cumulants $\kappa_{j}$ that

(2.7) $A_{n s}(t) \equiv n \mid(d / d t)^{\prime}\left[\sum_{r=1}^{3 k+3}(-1)^{r+1}\left\{\sum_{j=2}^{2 k+3} \mu_{j}\left(i t / n^{1 / 2}\right)^{j} / j !\right\}^{r} / r\right.$

$$
\left.-\sum_{r=2}^{2 k+3} \kappa_{j}\left(i t / n^{1 / 2}\right)^{j} / j !\right]
$$

$$
\leqslant C_{2}\left(1+t^{p}\right) t^{2(k+2)-l} n^{-(k+1)}
$$

for all $t>0$. Combining (2.5), (2.6) and (2.7), we see that if $0<t<\varepsilon n^{1 / 2}$ and $0 \leqslant l \leqslant 2 k+2$, then

$$
\begin{gathered}
\mid(d / d t)^{t}\left[n \log \alpha\left(t / n^{1 / 2}\right)-n\left\{\alpha\left(t / n^{1 / 2}\right)-1-\sum_{j=2}^{2 k+3} \mu_{j}\left(i t / n^{1 / 2}\right)^{j} / j !\right\}\right. \\
\left.-n \sum_{j=2}^{2 k+3} \kappa_{j}\left(i t / n^{1 / 2}\right)^{j} / j !\right] \mid \\
\leqslant A_{n 3}(t)+A_{n 4}(t)+A_{n 5}(t) \leqslant C\left(1+t^{p}\right) t^{2(k+2)-t} n^{-(k+1)} .
\end{gathered}
$$

Let

$$
A_{n 6}(t)=n\left\{\alpha\left(t / n^{1 / 2}\right)-1-\sum_{j=2}^{2 k+3} \mu_{j}\left(i t / n^{1 / 2}\right)^{j} / j !+\sum_{j=2}^{2 k+3} \kappa_{j}\left(i t / n^{1 / 2}\right)^{j} / j !\right\},
$$

and let $A_{n 7}(t)=n \log \alpha\left(t / n^{1 / 2}\right)-A_{n 6}(t)$. Then result (2.8) may be written in the form

$$
\left|(d / d t) A_{n 7}(t)\right| \leqslant C\left(1+t^{p}\right) t^{2(k+2)-t} n^{-(k+1)} .
$$


We may deduce from (2.6) that

$$
\left|(d / d t)^{l} A_{n 6}(t)\right| \leqslant C\left(1+t^{p}\right) .
$$

Let $\sum_{(l .1)}$ denote summation over vectors $\left(i_{1}, \ldots, i_{r}\right)$ with $1 \leqslant r \leqslant l, i_{a} \geqslant 1$ for each $a$, and $\sum_{a=1}^{r} i_{a}=l$. Let $\sum_{(l .2)}$ denote summation over vectors $\left(i_{1}, \ldots, i_{r}\right)$ and $\left(j_{1}, \ldots, j_{s}\right)$ with $0 \leqslant r \leqslant l-1,1 \leqslant s \leqslant l, i_{a} \geqslant 1$ and $j_{a} \geqslant 1$ for each $a$, and $\sum_{a=1}^{r} i_{a}+\sum_{a=1}^{s} j_{a}=l$. Combining (2.9) and (2.10), we see that

$$
\begin{aligned}
& \left.A_{n 8}(t) \equiv\left|(d / d t)^{\prime}\right| \alpha^{n}\left(t / n^{1 / 2}\right)-\exp \left\{A_{n 6}(t)\right\}\right] \mid \\
& \leqslant C_{1}\left(\sum_{(l .1)}\left|A_{n 6}^{\left(i_{1}\right)}(t) \cdot \ldots \cdot A_{n 6}^{\left(i_{i}\right)}(t)\right| \cdot\left|\exp \left\{A_{n 6}(t)\right\}\left[1-\exp \left\{A_{n 7}(t)\right\}\right]\right|\right. \\
& \quad+\sum_{(l .2)}\left|A_{n 6}^{\left(i_{1}\right)}(t) \cdot \ldots \cdot A_{n 6}^{\left(i_{1}\right)}(t) A_{n}^{\left(j_{j}\right)}(t) \cdot \ldots \cdot A_{n 7}^{\left(j_{j}\right)}(t)\right| \\
& \left.\cdot\left|\exp \left\{A_{n 6}(t)+A_{n 7}(t)\right\}\right|\right) \\
& \leqslant C_{2}\left(1+t^{p}\right)\left|\alpha^{n}\left(t / n^{1 / 2}\right)\right|\left[\left|1-\exp \left\{-A_{n 7}(t)\right\}\right|+t^{\left.2(k+2)-l_{n}-(k+1)\right] .}\right.
\end{aligned}
$$

In view of (2.9) (with $l=0$ ), there exists $\delta \in(0,1 / 2)$ such that $\left|A_{n 7}(t)\right| \leqslant C t$ whenever $0<t<n^{\delta}$ and $n$ is sufficiently large. For such values of $t$, we have

$$
\left|1-\exp \left\{-A_{n 7}(t)\right\}\right| \leqslant C_{1} t^{2(k+2)-l} \exp \left(C_{2} t\right) n^{-(k+1)} .
$$

An elementary argument shows that there exist $\varepsilon_{1}, \varepsilon_{2}>0$ such that

$$
\left|\alpha^{n}\left(t / n^{1 / 2}\right)\right| \leqslant \exp \left(-2 \varepsilon_{1} t^{2}\right)
$$

if $0<t \leqslant \varepsilon_{2} n^{1 / 2}$. Combining (2.11), (2.12) and (2.13), we see that if $0<t<n^{\delta}$ and $n$ is sufficiently large, then

$$
A_{n 8}(t) \leqslant C t^{2(k+2)-l} \exp \left(-\varepsilon_{1} t^{2}\right) n^{-(k+1)} .
$$

Next, observe that

$$
\begin{aligned}
A_{n 9}(t) \equiv & \left|(d / d t)^{l}\left[\exp \left\{A_{n 6}(t)\right\}-\exp \left\{n A_{n 1}(t)\right\}\left\{1+\sum_{j=1}^{2 k+1} P_{j}(i t) n^{-j / 2}\right\} e^{-t^{2} / 2}\right]\right| \\
\leqslant & \sum_{a=0}^{l}\left(\begin{array}{l}
l \\
a
\end{array}\right)\left|(d / d t)^{a} \exp \left\{n A_{n 1}(t)\right\}\right| \\
& \times\left|(d / d t)^{l-a}\right|\left[\exp \left\{n \sum_{j=3}^{2 k+3} \kappa_{j}\left(i t / n^{1 / 2}\right)^{j} / j !\right\}\right. \\
& \left.\left.-\left\{1+\sum_{j=1}^{2 k+1} P_{j}(i t) n^{-j / 2}\right\}\right] e^{-t^{2} / 2}\right) \mid
\end{aligned}
$$


Now,

$$
\begin{aligned}
\exp \left\{n \sum_{j=3}^{2 k+3} \kappa_{j}\left(i t / n^{1 / 2}\right)^{j} / j !\right\}= & +\sum_{j=1}^{2 k+1} P_{j}(i t) n^{-j / 2} \\
& +\sum_{r=2 k+2}^{\infty}\left\{n \sum_{j=3}^{2 k+3} \kappa_{j}\left(i t / n^{1 / 2}\right)^{j} / j !\right\} / r \\
& +n\left(i t / n^{1 / 2}\right)^{2(k+2)} A_{n, 10}(t),
\end{aligned}
$$

where $A_{n, 10}(t)$ denotes a polynomial in it of degree not exceeding $(2 k+1)$ $\times(2 k+3)-2(k+2)$, and all of whose coefficients are uniformly bounded. Therefore,

$$
\begin{aligned}
A_{n, 11}(t) \equiv & \left|(d / d t)^{l}\left[\exp \left\{n \sum_{j=3}^{2 k+3} \kappa_{j}\left(i t / n^{1 / 2}\right)^{j} / j !\right\}-\left\{1+\sum_{j=1}^{2 k+1} P_{j}(i t) n^{-j / 2}\right\}\right]\right| \\
& \leqslant \sum_{r=2 k+2}^{\infty}\left|(d / d t)^{l}\left\{n \sum_{j=3}^{2 k+3} \kappa_{j}\left(i t / n^{1 / 2}\right)^{j} / j !\right\}\right| / r ! \\
& +C\left(1+t^{p}\right) t^{2(k+2)-l_{n}-(k+1)} .
\end{aligned}
$$

The series on the right hand side is dominated by $C\left(1+t^{p}\right) t^{6(k+1)} n^{-(k+1)}$ uniformly in $0<t<n^{1 / 2}$. Consequently,

$$
A_{n, 11}(t) \leqslant C\left(1+t^{p}\right) t^{2(k+2)-l} n^{-(k+1)} .
$$

It follows from (2.2) and (2.4) that if $\varepsilon \in(0,1]$ is sufficiently small, and if $0<t<\varepsilon n^{1 / 2}$, then

$$
\left|(d / d t)^{l} \exp \left\{n A_{n 1}(t)\right\}\right| \leqslant C e^{-t^{2} / 4}
$$

Substituting (2.16) and (2.17) into (2.15), we see that if $0<t<\varepsilon n^{1 / 2}$, then

$$
A_{n 9}(t) \leqslant C t^{2(k+2)-l} \exp \left(-t^{2} / 5\right) n^{-(k+1)} .
$$

Since

$$
\left|(d / d t)^{l}\left[\left\{1+\sum_{j=1}^{2 k+1} P_{j}(i t) n^{-j / 2}\right\} e^{-t^{2} / 2}\right]\right| \leqslant C\left(1+t^{p}\right) e^{-t^{2} / 2}
$$


it follows that

$$
\begin{aligned}
A_{n, 12}(t) \equiv \mid(d / d t)^{l}[ & \exp \left\{n A_{n 1}(t)\right\}\left\{1+\sum_{j=1}^{2 k+1} P_{j}(i t) n^{-j / 2}\right\} e^{-t^{2} / 2} \\
& \left.-\left\{1+n A_{n 1}(t)\right\}\left\{1+\sum_{j=1}^{2 k+1} P_{j}(i t) n^{-j / 2}\right\} e^{-t^{2} / 2}\right] \mid \\
\leqslant & C_{1}\left(1+t^{p}\right) e^{-t^{2} / 2} \sum_{r=2}^{\infty}(r !)^{-1} \sum_{a=0}^{l}\left|(d / d t)^{a}\left\{n A_{n 1}(t)\right\}^{r}\right|
\end{aligned}
$$

Now,

$$
\begin{aligned}
A_{n, 13}(t) \equiv & \sum_{a=0}^{l}\left|(d / d t)^{a}\left\{n A_{n 1}(t)\right\}^{r}\right| \\
\leqslant & C^{l}\left\{\left|n A_{n 1}(t)\right|^{r-2}+\left|n A_{n 1}(t)\right|^{\max (0, r-2-l)}\right\} \\
& \times\left[\left|n A_{n 1}(t)\right|^{2}+\left|n A_{n 1}(t)\right| \sum_{a=1}^{l}\left|n A_{n 1}^{(a)}(t)\right|\right. \\
& \left.\quad+\sum_{a=2}^{l} \sum_{(a .3)}\left|n A_{n 1}^{\left(j_{1}\right)}(t) \cdot \ldots \cdot n A_{n 1}^{\left(j_{2}\right)}(t)\right|\right],
\end{aligned}
$$

where $C$ does not depend on $r$, and where $\sum_{(\mathbf{a} \cdot 3)}$ denotes summation over vectors $\left(j_{1}, \ldots, j_{s}\right)$ with $2 \leqslant s \leqslant a$, each $j_{b} \geqslant 1$, and $\sum_{b=1}^{s} j_{b}=a$. Using (2.3) and (2.4), we see that if $0<t<\varepsilon n^{1 / 2}$, and if $\varepsilon$ is sufficiently small, then

$$
A_{n, 13}(t) \leqslant C r^{l}\left\{\left(t^{2} / 10\right)^{r-2}+\left(t^{2} / 10\right)^{\max (0, r-2-l)}\right\}\left(1+t^{p}\right) t^{2 k+3-l} \delta_{n}^{2},
$$

where $C$ does not depend on $r$. Substituting into (2.19), we obtain

$$
A_{n, 12}(t) \leqslant C t^{2 k+3-t} \exp \left(-t^{2} / 4\right)_{k} \delta_{n}^{2} .
$$

In view of (2.3), we have

$$
\begin{gathered}
A_{n, 14}(t) \equiv \mid(d / d t)^{l}\left[\left\{1+n A_{n 1}(t)\right\}\left\{1+\sum_{j=1}^{2 k+1} P_{j}(i t) n^{-j / 2}\right\} e^{-t^{2} / 2}\right. \\
\left.-\left\{1+\sum_{j=1}^{2 k+1} P_{j}(i t) n^{-j / 2}+n A_{n 1}(t)\right\} e^{-t^{2} / 2}\right] \mid \\
\leqslant C\left(1+t^{p}\right) t^{2 k+3-l} \exp \left(-t^{2} / 2\right) n^{-1 / 2},
\end{gathered}
$$

uniformly in $t$. (Note that the polynomials $P_{j}(i t)$ satisfy $P_{j}(0)=0$.) Combining (2.14), (2.18), (2.20) and (2.21), we see that if $0<t<n^{\delta}$, and if $n$ is sufficiently 
large, then

$$
\begin{aligned}
\left|{ }_{k} d_{n}^{(l)}(t)\right| & \leqslant A_{n 8}(t)+A_{n 9}(t)+A_{n, 12}(t)+A_{n, 14}(t) \\
& \leqslant C t^{2 k+3-t} \exp \left(-\varepsilon t^{2}\right)\left(n^{-(k+1)}+{ }_{k} \delta_{n}^{2}+n^{-1 / 2}{ }_{k} \delta_{n}\right) .
\end{aligned}
$$

To treat the case where $t>n^{\delta}$, we observe that

$$
\begin{aligned}
\left|{ }_{k} d_{n}^{(l)}(t)\right| \leqslant & \left|(d / d t)^{l} \alpha^{n}\left(t / n^{1 / 2}\right)\right| \\
& +\left|(d / d t)^{l}\left[\left\{1+\sum_{j=1}^{2 k+1} P_{j}(i t) n^{-j / 2}+n A_{n 1}(t)\right\} e^{-t^{2} / 2}\right]\right| \\
& \leqslant C_{1}\left(1+t^{p}\right) n^{p}\left\{\left|\alpha\left(t / n^{1 / 2}\right)\right|^{n-l}+e^{-t^{2} / 2}\right\}
\end{aligned}
$$

for all $t>0$. It follows from (2.13) that for some $\varepsilon \in(0,1 / 4)$ and $\eta>0$, we have $\left|\alpha\left(t / n^{1 / 2}\right)\right|^{n-l} \leqslant C \exp \left(-2 \varepsilon t^{2}\right)$ whenever $0<t<\eta n^{1 / 2}$. Consequently, $\left|{ }_{k} d_{n}^{(l)}(t)\right|$ $\leqslant C t^{2 k+3-l} \exp \left(-\varepsilon t^{2}\right) n^{-(k+1)}$ uniformly in $n^{\delta}<t<\eta n^{1 / 2}$. Therefore (2.22) holds uniformly in $0<t<\eta n^{1 / 2}$.

Let

$$
\rho=\sup _{t>\eta}|\alpha(t)|<1 .
$$

If $\eta n^{1 / 2}<t<n^{k+1}$, then it follows from (2.23) that

$$
\left|{ }_{k} d_{n}^{(l)}(t)\right| \leqslant C_{2}\left(1+t^{p}\right) n^{p}\left(\rho^{n}+e^{-t^{2} / 2}\right)<C_{3} r^{n}
$$

for some $r<1$.

Returning to (2.1), taking $T=n^{k+1}$, and estimating $\left|{ }_{k} d_{n}^{(l)}(t)\right|$ using (2.22) if $0<t<\eta n^{1 / 2}$, or using (2.24) if $\eta n^{1 / 2}<t<n^{k+1}$, we see that

$$
\left.\sup _{-\infty<x<\infty}\left(1+|x|^{2 k+2}\right)\right|_{k} D_{n}(t) \mid=O\left(n^{-(k+1)}+{ }_{k} \delta_{n}^{2}+n^{-1 / 2} \delta_{k}\right),
$$

which proves Theorem 1 .

\section{Acknowledgment}

The second author expresses his gratitude to the Australian National University, in particular to the Department of Statistics in the Faculty of Economics and Commerce, for the award of an Honorary Research Fellowship during the tenure of which this work was undertaken. 


\section{References}

[1] P. Hall, Rates of convergence in the central limit theorem (Pitman, London, 1982).

[2] P. Hall, 'A leading term approach to asymptotic expansions in the central limit theorem', Proc. London Math. Soc. 49 (1984), 423-444.

[3] C. C. Heyde and T. Nakata, 'On the asymptotic equivalence of $L_{p}$ metrics for convergence to normality', Z. Wahrsch. Verw. Gebiete 68 (1984), 97-106.

[4] V. V. Petrov, Sums of independent random variables (Springer-Verlag, Berlin, 1975).

Department of Statistics

Faculty of Economics and Commerce

Australian National University

G.P.O. Box 4

Canberra, A.C.T. 2601

Australia
Department of Mathematics

Chukyo University 101-2 Yagota Honmachi Showa-ku, Nagoya 468

Japan 